\title{
Über die Pyruvat-Decarboxylase-Aktivität von E. coli unter verschiedenen Wachstumsbedingungen
}

\author{
THEODOR GÜNTHER
}

\author{
Physiologisch-Chemisches Institut der Freien Universität Berlin \\ (Z. Naturforschg. 24 b, 428-432 [1969]; eingegangen am 6. September 1968)
}

\begin{abstract}
Uber Nacht auf Hexosen gewachsene Zellen von E. coli haben eine hohe Pyruvat-DecarboxylaseAktivität mit einem Hill-Exponenten von 1,9. Zellen, die auf anderen Substraten bzw. unter $\mathrm{Mg}-\mathrm{PO}_{4}$ - oder $\mathrm{NH}_{4}$-Mangelbedingungen gewachsen sind, weisen eine niedrige Pyruvat-Decarboxylase-Aktivität auf, ihr $\mathrm{H}$ ill - Exponent beträgt 0,9 .

Während der log-Phase sinkt die Pyruvat-Decarboxylase-Aktivität auf $2-5 \%$ des Ausgangswertes und steigt am Ende des Wachstums in glucosehaltigen Medien wieder an.

Die Ergebnisse werden als (vorübergehende) während der log-Phase auftretende KatabolitRepression des durch Hexosen bzw. deren Abbauprodukte induzierbaren Enzyms gedeutet.
\end{abstract}

In früheren Versuchen konnten wir zeigen, da $\beta$ Zellen von E. coli, die unter verschiedenen Mangelbedingungen gewachsen waren, einige Enzyme in unterschiedlicher Menge bilden ${ }^{1,2}$. In der PyruvatDecarboxylase fanden wir ein weiteres Enzym, dessen Aktivität in der Zelle von den Wachstumsbedingungen abhängt.

\section{Methode}

Die Bakterien wurden, wie an anderer Stelle ausführlich beschrieben ${ }^{3}$, gezüchtet. Die $\mathrm{Mg}$-Konzentration im Medium betrug bei den Kontrollzellen $10^{-3} \mathrm{M}$, bei den Mg-armen Zellen $3 \cdot 10^{-6} \mathrm{M}$. Die Zusammensetzung der $\mathrm{K}$-, $\mathrm{SO}_{4}$, $\mathrm{PO}_{4^{-}}$und $\mathrm{NH}_{4}$-armen Medien wurde bereits mitgeteilt ${ }^{4}$.

Die über Nacht gewachsenen Zellen wurden einmal mit bidest. Wasser gewaschen, in kalter $0,15 \mathrm{M} \mathrm{KCl}$ aufgenommen und unter Kühlen in einer Eis-KochsalzMischung mit Ultraschall aufgeschlossen.

Die Decarboxylierung des Pyruvats wurde bei $37^{\circ}$ manometrisch bestimmt. Der Ansatz enthielt: $1 \mathrm{ml}$ Homogenat bzw. $1 \mathrm{ml}$ Überstand, $1 \mathrm{ml} \mathrm{0,1} \mathrm{M}$ Phosphatpuffer $\mathrm{pH}$ 6,0 (in einigen Versuchen $1 \mathrm{ml}$ 0,2 $\mathrm{m}$ Imidazolpuffer $\mathrm{pH}$ 6,0), $0,2 \mathrm{ml} 1,2 \mathrm{M}$ Na-Pyruvat, $0,1 \mathrm{ml}$ $1,2 \cdot 10^{-2} \mathrm{M}$ Thiamin-pyrophosphat und $0,1 \mathrm{ml} 2,4 \cdot 10^{-2}$ $\mathrm{M} \mathrm{MgCl}_{2}$. Durch $10^{-3} \mathrm{M}$ Mg wird die Pyruvat-Decarb. oxylase nur geringfügig erhöht und durch $10^{-2} \mathrm{M}$ EDTA in Übereinstimmung mit dem Verhalten der Hefe-Pyruvat-Decarboxylase nicht signifikant geändert ${ }^{5}$. Der Proteingehalt der Ansätze wurde nach LOwRY et al. ${ }^{6}$ ermittelt.

1 Th. Günther u. P. Mariss, Z. Naturforschg. 23 b, 338 [1968].

2 Th. Günther u. P. Mariss, Hoppe Seyler's Z. physiol. Chem. 349, 623 [1968].

3 Th. Günther u. F. Dorn, Z. Naturforschg. 21 b, 1076 [1966].

\section{Ergebnisse}

\section{Kinetik der Pyruvat-Decarboxylase}

Die Pyruvat-Decarboxylase-Aktivität von E.coli zeigt einen S-förmigen Verlauf, wenn man sie als Funktion der Substratkonzentration $[S]$ darstellt [Abb. 1, Kurve $\mathrm{I}_{0}$ ). Bei der Darstellung nach Hill ${ }^{7}$ $\left(\log \frac{v}{V \max -v}\right.$ als Funktion von $\left.\log [\mathrm{S}]\right)$ erhält man für $n$ den Wert 1,9 [Abb. 2, I]. Wird das Homogenat oder der $100000 \mathrm{~g}$ Überstand $10 \mathrm{Min}$. auf $55{ }^{\circ} \mathrm{C}$ erhitzt oder in Gegenwart von $10^{-4} \mathrm{M} \mathrm{Hg} \mathrm{Cl} 2$ getestet, dann mißt man nur noch eine geringe Aktivität und die S-förmige Kurve geht in eine einfache Michaelis-Menten-Kinetik über [Abb. 1, Kurve I]. Dabei nimmt $n$ den Wert 0,9 an [Abb. 2, Kurve II].

\section{Pyruvat-Decarboxylase-Aktivität von Mangelzellen}

Über Nacht in Mg-armen Medien gewachsene Zellen besitzen nur noch ca. 5\% der Pyruvat-Decarboxylase-Aktivität der Kontrollzellen [Tab. 1]. Während bei den Kontrollzellen die spezifische Aktivität der Pyruvat-Decarboxylase im Uberstand nach Zentrifugation ansteigt, nimmt sie bei Mg-arm gewachsenen Zellen im Überstand ab.

Bei der Darstellung der Pyruvat-DecarboxylaseAktivität Mg-armer Zellen als Funktion von $[S]$ er-

${ }^{4}$ Th. GÜnther u. P. Mariss, Z. Naturforschg. 23 b, 334 [1968].

5 A. Schellenberger, Angew. Chem. 79, 1050 [1967].

${ }_{6}^{6}$ O. H. Lowry, N. J. Rosebrough, A. L. Farr u. R. J. Randall, J. biol. Chemistry 193, 265 [1951].

7 W. E. L. Brown u. A. V. Hill, Proc. Roy. Soc. [London], Ser. B 94, 297 [1922]. 


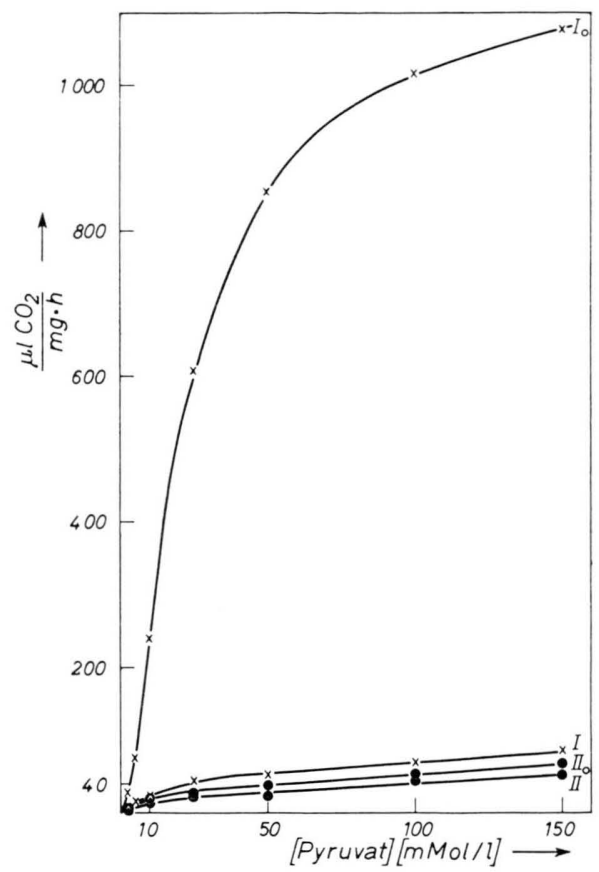

Abb. 1. Pyruvat-Decarboxylase-Aktivität der Zellhomogenate in Abhängigkeit von der Pyruvat-Konzentration. $\mathrm{I}_{0}$ Mg-reich gewachsene E. coli, I Homogenat aus Mg-reich gew. Zellen, $10 \mathrm{Min}$. auf $55^{\circ}$ erwärmt. $\mathrm{II}_{0} \mathrm{Mg}$-arm gewachsene $E$. coli, II Homogenat aus Mg-arm gew. Zellen, 10 Min. auf $55^{\circ}$ erwärmt.

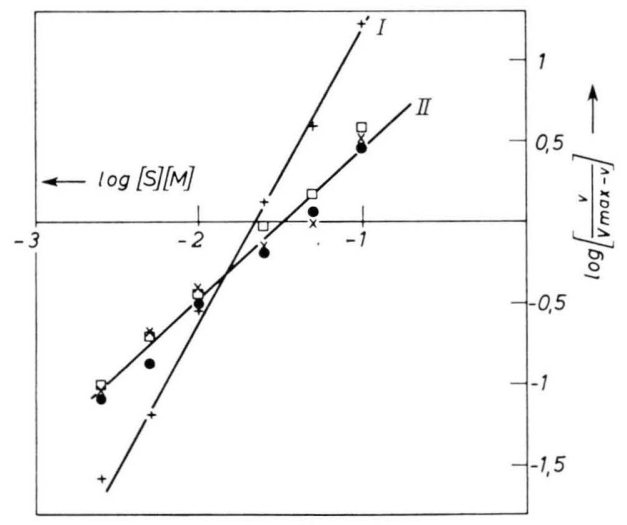

Abb. 2. Darstellung der Gleichung $\log$ $V \max -v=$

- log K. + Homogenat aus Mg-reich gewachsenen Zellen. $\square$ Homogenat aus Mg-reich gewachsenen Zellen nach 10 Min. Erwärmen auf $55^{\circ}, \times$ Homogenat aus Mg-arm gewachsenen Zellen, - Homogenat aus Mg-arm gewachsenen Zellen nach 10 Min. Erwärmen auf $55^{\circ}$.

hält man eine Michaelis-Menten-Kinetik [Abb. $\left.1, \mathrm{II}_{0}\right]$. Aus der Hill-Darstellung ermittelt man bei Mg-armen Zellen für $n$ einen Wert von 0,9. Erhitzt man das Homogenat aus Mg-armen Zellen 10 Min. auf $55^{\circ}$, so findet man danach eine gerin-

\begin{tabular}{lccc}
\hline & & \multicolumn{2}{c}{ Úberstand } \\
& Homogenat & $3000 \mathrm{~g}$ & $100000 \mathrm{~g}$ \\
\hline Mg-reich & 805 & 1080 & 1620 \\
Mg-arm & 39,5 & 16,7 & 10 \\
\hline
\end{tabular}

Tab. 1. Pyruvat-Dekarboxylase-Aktivität [in $\mu \mathrm{l} \quad \mathrm{CO}_{2} / \mathrm{mg}$ Prot. Stde.] in den Homogenaten, $3000 \mathrm{~g}$ - und $100000 \mathrm{~g}$ Überständen von $\mathrm{Mg}$-reich und Mg-arm gewachsenen E. coli.

gere Abnahme der Enzymaktivität [Abb. 1, II]. Der Hill-Exponent $n$ beträgt bei den erhitzten Mgarmen Zellen ebenfalls 0,9 [Abb., 2, II] .

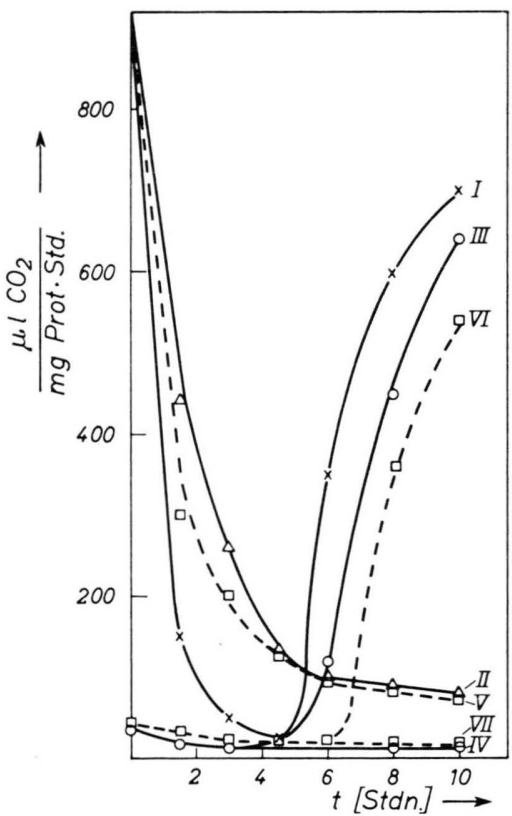

Abb. 3. Pyruvat-Decarboxylase-Aktivität während des Wachstums in verschiedenen Medien. Die Zellen wurden zu den angegebenen Zeiten entnommen, abzentrifugiert, einmal in bidest. Wasser gewaschen und eingefroren. Zur manometrischen Bestimmung der Pyruvat-Decarboxylase-Aktivität wurden sie in $0.1 \mathrm{M} \mathrm{KCl} \mathrm{mit} \mathrm{Ultraschall} \mathrm{homogenisiert.} \mathrm{Die} \mathrm{Glucose-}$ konzentration und der $\mathrm{pH}$-Wert der Medien wurden während des Wachstums bei $37{ }^{\circ} \mathrm{C}$ mit Indikator-Papieren kontrolliert und durch Zugabe von Glucose und $\mathrm{NaHCO}_{3}$ nachgestellt. I. Mg-reich gewachsene Zellen in Normalmedium. II. Mg-reich gewachsene Zellen in Mg-freiem Medium. III. Mg-arm gewachsene Zellen in Normalmedium. IV. Mg-arm gewachsene Zellen in $10^{-3} \mathrm{M}$ Mg-haltigem Medium ohne Aminosäuren und $\mathrm{NH}_{4}$. V. Mg-reich gewachsene Zellen in $\mathrm{PO}_{4}$-freiem Medium. VI. $\mathrm{PO}_{4}$-arm gewachsene Zellen in Normalmedium. VII. $\mathrm{PO}_{4}$-arm gewachsene Zellen in Aminosäuren- und $\mathrm{NH}_{4}$ freiem Medium.

\section{Spezifität}

Aus $\alpha$-Ketoglutarsäure wird von den Kontrollzellen nur $1-2 \%$ der $\mathrm{CO}_{2}$-Menge freigesetzt, die unter gleichen Bedingungen aus Pyruvat abgespalten wird. 
Durch 10 Min. Erwärmen auf $55^{\circ}$ wird die Decarboxylierung von $\alpha$-Ketoglutarsäure nur wenig vermindert. Bei Mg-arm gewachsenen Zellen ist die $\mathrm{CO}_{2}$-Freisetzung aus $\alpha$-Ketoglutarsäure unmeßbar niedrig.

Die geringe $\mathrm{CO}_{2}$-Abspaltung aus Pyruvat ist nicht spezifisch für Zellen, die unter Mg-Mangel gewachsen sind. $\mathrm{PO}_{4}$ - oder $\mathrm{NH}_{4}$-arm gewachsene Zellen hatten ebenfalls eine niedrige Pyruvat-Decarboxylase-Aktivität, die sich nach 10 Min. Erwärmen auf $55^{\circ}$ und in der $\mathrm{H}$ ill - Darstellung wie die von Mg-armen Zellen verhielt. Bei Zellen, die unter $\mathrm{K}$ - oder $\mathrm{SO}_{4}$-Mangelbedingungen gewachsen waren, fanden wir dagegen eine unverändert hohe Pyruvat-DecarboxylaseAktivität [Tab.2].

\begin{tabular}{cccc}
\hline K-arm & $\mathrm{SO}_{4}$-arm & $\mathrm{PO}_{4}$-arm & $\mathrm{NH}_{4}$-arm \\
\hline 820 & 800 & 44 & 58 \\
\hline
\end{tabular}

Tab. 2. Pyruvat-Decarboxylase-Aktivität in den Homogenaten von $\mathrm{K}$-arm, $\mathrm{SO}_{4}$-arm, $\mathrm{PO}_{4}$-arm und $\mathrm{NH}_{4}$-arm gewachsenen E. coli $[\mu \mathrm{l} \mathrm{CO} 2 / \mathrm{mg}$ Prot. Stde. $]$.

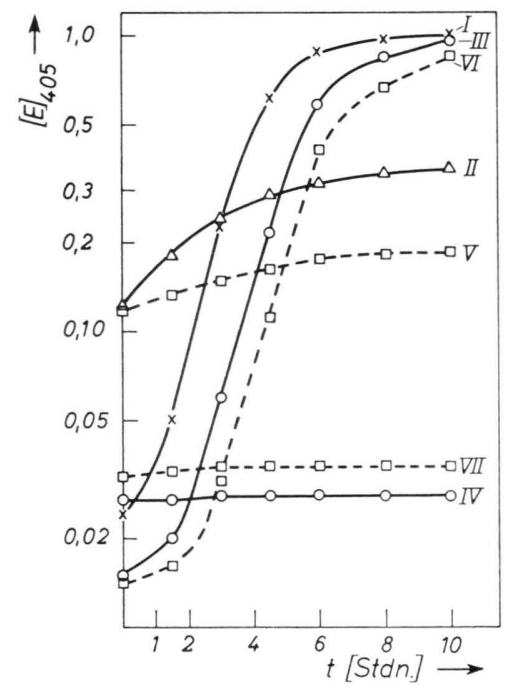

Abb. 4. Wachstum von E. coli in verschiedenen Medien. Die Wachstumskurven entsprechen den Versuchen in den Abbn. 3 und 5. Das Wachstum wurde durch Extinktionsmessung bei $405 \mathrm{~nm}$ bestimmt.

Die Pyruvat-Decarboxylase-Aktivität ist außerdem abhängig vom Substrat des Wachstumsmediums. Auf Hexosen gewachsene Bakterien besaßen die höchste Aktivität, gefolgt von Glycerin. Die auf den übrigen Substraten gewachsenen Zellen hatten eine niedrige Decarboxylase-Aktivität [Tab. 3] und einen H ill - Exponenten von 0,9.

\begin{tabular}{|c|c|c|}
\hline Substrat & Mg-reich & Mg-arm \\
\hline Glycerin & 420 & 49 \\
\hline Pyruvat & 96 & 91 \\
\hline Lactat & 48 & 47 \\
\hline Fructose & 735 & \\
\hline Galaktose & 820 & \\
\hline Mannose & 730 & \\
\hline Citrat & 90 & \\
\hline$\alpha$-Ketoglutarat & 14 & \\
\hline Succinat & 58 & \\
\hline Alanin & 78 & \\
\hline Asparaginat & 51 & \\
\hline Glutamat & 51 & \\
\hline
\end{tabular}

Tab. 3. Pyruvat-Decarboxylase-Aktivität $\left[\mu \mathrm{l} \mathrm{CO}_{2} / \mathrm{mg}\right.$ Prot. Stde.] in den Homogenaten von $E$. coli, die über Nacht in Wachstumsmedien mit verschiedenen Substraten [Konzentration jeweils $0,5 \%]$ gezüchtet wurden.

\section{Pyruvat-Decarboxylase-Aktivität während des Wachstums}

Inkubiert man über Nacht in glucosehaltigem Medium gewachsene Kontrollzellen mit hoher PyruvatDecarboxylase in normalem Wachstumsmedium, dann nimmt die spezifische Aktivität des Enzyms während der Wachstumsphase unabhängig von der C-Quelle des Mediums stark ab, erst wenn die Zellen aufhören zu wachsen und Glucose im Medium vorhanden ist, steigt sie wieder an [Abbn. 3, 4, Kurve I]. Läßt man Mg-arm [Kurve III] oder $\mathrm{PO}_{4}$. arm [VI] gewachsene Zellen in normalem Medium (mit Glucose) wachsen, dann steigt ihre von vornherein niedrige Pyruvat-Decarboxylase ebenfalls

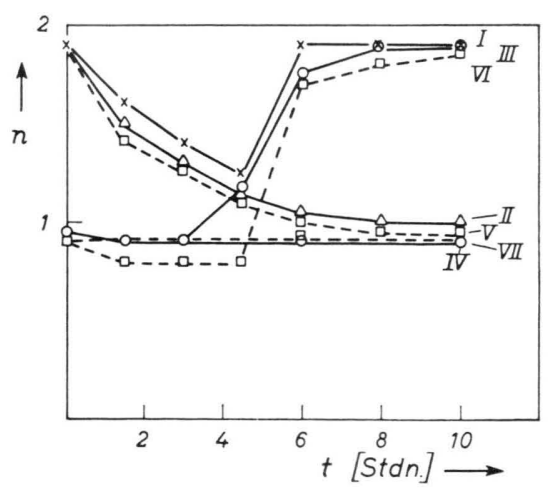

Abb. 5. Änderung des Hill-Exponenten während des Wachstums in verschiedenen Medien (s. Leg. Abb. 3).

erst an, wenn die logarithmische Wachstumsphase zu Ende geht. Wachsen Bakterien mit hoher PyruvatDecarboxylase-Aktivität in Mg-freiem [Kurve II] oder $\mathrm{PO}_{4}$-freiem [V] Medium weiter, wobei es noch zu einer geringen Zellvermehrung kommt, sinkt ihre 
Decarboxylase-Aktivität sehr schnell auf den für diese Zellen charakteristischen niedrigen Wert ab.

Die Zunahme der Decarboxylase-Aktivität erfolgt nur, wenn Proteinsynthese möglich ist. Bringt man Mg-arm bzw. $\mathrm{PO}_{4}$-arm gewachsene Zellen in ein Mg-haltiges [Kurve IV] bzw. $\mathrm{PO}_{4}$-haltiges [VII] Medium ohne Aminosäuren und Stickstoffquelle, so bleibt die Pyruvat-Decarboxylase auf ihrem niedrigen Niveau.

Das Absinken und Ansteigen der Pyruvat-Decarboxylase-Aktivität ist mit einer gleichsinnigen Änderung des Hill-Exponenten verbunden [Abb. 5].

\section{Diskussion}

In E. coli gibt es verschiedene Enzyme, die $\mathrm{CO}_{2}$ aus Pyruvat freisetzen und zwar einen Liponsäureund FAD-haltigen Multienzymkomplex [E.C. 1.2.4.1] bestehend aus Pyruvat-Decarboxylase, Lipoylreduktase-Transacetylase und DihydrolipoylDehydrogenase $^{8,9}$ sowie verschiedene DPN-, CoAund Liponsäure-unabhängige Enzyme ${ }^{9,10}$ [E.C. 1.2.2.2, 1.2.3.3, 4.1.1.1].

Das unter den gewählten Bedingungen erfaßte Enzym benötigte kein DPN, CoA, $\mathrm{O}_{2}, \mathrm{PO}_{4}$ und keine Liponsäure. Außer $\mathrm{CO}_{2}$ wurde kein weiteres Gas freigesetzt. Als Endprodukt der Reaktion fanden wir Acetoin [Bestimmung nach WESTERFELD ${ }^{11}$ ]. Das Enzym entsprach auch in seinem Verhalten gegenüber $\mathrm{Mg}$ und EDTA sowie in seinem pH-Optimum [bei $\mathrm{pH} 5,5-6$ ], seiner $K m$ für Thiaminpyrophosphat $\left[K \mathrm{~m}=3 \cdot 10^{-6} \mathrm{M}\right]$ und seiner halbmaximalen Aktivität mit Pyruvat $\left[3 \cdot 10^{-2} \mathrm{M}\right]$ der nichtoxydativen Pyruvat-Decarboxylase aus Hefe [E.C. 4.1.1.1 $]^{10}$.

Außerdem ermittelten wir auch bei kristallisierter Hefepyruvat-Decarboxylase [E.C. 4.1.1.1., Fa. Boehringer] [im optischen Test mit Alkoholdehydrogenase] einem etwa gleich großen H ill - Exponenten $[n=1,7]$.

Die S-förmige Kinetik und der Hill-Exponent von 1,9 erinnern an einen positiven homotropen co-

${ }^{8}$ L. J. Reed u. D. J. Cox, Ann. Rev. Biochem. 35, 57 [1966].

9 D. R. SANAdi, in: The Enzymes, 7, 307 [1963], Hrsg. P. D. Boyer, W. Lardy u. K. Myrbäck, Acad. Press, New York 1963.

10 M. F. Utter, in: The Enzymes, 5, 319 [1961], Hrsg. P. D. Boyer, W. Lardy u. K. Myrbäck, Acad. Press, New York 1961. operativen Effekt, der für die Kinetik allosterischer Enzyme typisch ist. Die Erniedrigung des HillExponenten auf 0,9 durch Hitzebehandlung, die wir auch bei kristallisierter Hefepyruvat-Decarboxylase fanden, könnte der "Desensibilisierung“ allosterischer Enzyme entsprechen. Es ist uns jedoch nicht gelungen, einen für allosterische Enzyme charakteristischen Effektor nachzuweisen, so daß eine Zuordnung des Enzyms zu den allosterischen Enzymen fraglich ist, denn eine S-förmige Kinetik ergibt sich auch, wenn ein Enzym mehrere Substratmoleküle bindet ${ }^{12}$, aus mehreren Untereinheiten besteht ${ }^{13}$, oder wenn [ohne cooperativen Effekt] für die enzymatische Reaktion mehrere Reaktionswege existieren ${ }^{14}$.

Die Abnahme der Pyruvat-Decarboxylase bei den Mangelzellen und während der log-Phase des Wachstums ist nicht auf einen Inhibitor zurückzuführen. Wenn man die Homogenate aus Decarboxylase-reichen und Decarboxylase-armen Zellen mischt, erhält man eine Aktivität, die dem arithmetischen Mittel entspricht. Ein Mg-Mangel des Enzyms ist auch auszuschließen, da die Bestimmung in Gegenwart von Mg erfolgte.

Die Abnahme der spezifischen Aktivität der Pyruvat-Decarboxylase während der log-Phase kommt zustande, weil während dieser Zeit keine Nettosynthese des Enzyms erfolgt. Als Ursachen kämen (vorübergehende) Katabolit-Repression oder eine unterbrochene Induktion in Frage. Weil die Enzymaktivität beim Wachsen unter Mangelbedingungen stärker abnimmt als der Zellvermehrung entspricht, kann die Enzymaktivität unter Mangelbedingungen wahrscheinlich noch zusätzlich verringert werden. Da an der niedrigen Decarboxylase-Aktivität von Mangelzellen ein $\mathrm{H}$ ill - Exponent von 0,9 ermittelt wurde, kann man diskutieren, ob die geringe Restaktivität von einem anderen decarboxylierenden Enzym stammt. Hierfür spräche, daß wir bei der niedrigen Decarboxylase-Aktivität Mg-armer Zellen kein Acetoin nachweisen konnten. Eine Entscheidung ist erst nach Isolierung der Enzyme möglich.

11 W. W. Westerfeld, J. biol. Chemistry 161, 495 [1945].

12 B.D. Sanwal, C.S.STACHOW u. R. A. CoOK, Biochemistry 4, 410 [1965].

13 J. Monod, J. Wyman u. J. P. Changeux, J. molecular Biol. 12, 88 [1965].

14 J. R. Sweeny u. J. R. Fisher, Biochemistry 7, 561 [1968]. 
Durch die Aktivitätsabnahme während des Wachstums unterscheidet sich das erfaßte Enzym ebenfalls von der Pyruvat-Decarboxylase des MultienzymKomplexes [E.C. 1.2.4.1], deren Aktivität während des Wachstums konstant bleibt ${ }^{15}$ oder sogar etwas ansteigt ${ }^{16}$. Eine ähnliche Aktivitäts-Abnahme während des Wachstums wurde auch beim Pyruvatoxydase- [E.C. 1.2.2.2] und phosphorklastischen System von $E$. coli gefunden ${ }^{15}$.
Die Beziehungen der Pyruvat-Decarboxylase zur C-Quelle im Wachstumsmedium lassen sich mit einer Induktion des Enzyms durch abbaubare Hexosen bzw. eines ihrer Abbauprodukte erklären. Diese Induktion erfolgt aber erst, wenn die Bakterien das Ende ihrer log-Phase erreichen.

15 M. O. Oster u. L. P. Hager, Bacteriol. Proc. 1964, 101.

16 U. Henning, C. Herz u. K. Szolyvay, Z. Vererbungsl. 95 , 236 [1964].

Über Proteine aus Tumor- und Normalgewebe

\title{
7. Zur elektrophoretischen Charakterisierung basischer Proteine in cytoplasmatischen Säureextrakten aus Rattenhepatom und normaler Rattenleber*
}

\author{
K.-D. SCHWENKE \\ Institut für Ernährung der Deutschen Akademie der Wissenschaften zu Berlin, \\ Potsdam-Rehbrücke \\ (Z. Naturforschg. 24 b, 432—435 [1969] ; eingegangen am 26. August 1968)
}

\begin{abstract}
Ungepufferte Lösungen von $\mathrm{KCl} / \mathrm{KOH}$ eignen sich für die elektrophoretische Trennung säureextrahierter, cytoplasmatischer Organproteine. Mit Hilfe der freien Elektrophorese im System 0,05-m. KCl/KOH wurde der isoelektrische Punkt einer basischen Leberproteinfraktion (h-Protein) bei $\mathrm{pH}$ 8,3-8,4 bestimmt. Diese Fraktion ist in den Extrakten aus Hepatom Berlin und DAENAHepatom nur schwach vertreten oder fehlt ganz.

Mit Hilfe der Stärkegel-Elektrophorese in 0,01-n. HCl wurden die säurelöslichen Cytoplasmaproteine aus Rattenleber und 2 Hepatomen in 5-6 scharfe Zonen getrennt.
\end{abstract}

Elektronenphoretische Methoden wurden wiederholt zu vergleichenden Untersuchungen von Organproteinen aus Tumor- und Normalgeweben herangezogen ${ }^{1-4}$. Dabei konnten charakteristische Unterschiede besonders bei den basischen Cytoplasmaproteinen, die durch Säureextraktion angereichert wurden, festgestellt werden ${ }^{5}$. Die Verwendung konventioneller Puffersysteme zur freien Elektrophorese der säureextrahierten Proteine ergab zwar eine relativ gute Trennung der Hauptfraktionen, führte aber zur Ausfällung eines hohen Proteinanteils.

In der vorliegenden Arbeit wird über eine verbesserte Auftrennung säureextrahierter, cytoplasmatischer Proteine aus normaler Rattenleber und zwei Hepatomen und die Charakterisierung der basischen Hauptfraktion mit Hilfe der Elektrophorese in ungepufferten Elektrolytlösungen berichtet.

1 S. Sorof u. P. P. Cohen, Cancer Res. 11, 376 [1951].

2 S. Sorof, P. P. Cohen, E. C. Miller u. J. A. Miller, Cancer Res. 11, 383 [1951].

${ }^{3}$ K. D. Schwenke, Z. Krebsforsch. 68, 112 [1966].

${ }^{4}$ K. D. Schwenke, R. Plass u. M. Kujawa, Z. Krebsforsch. 68, 234 [1966].

\section{Experimenteller Teil}

Als Ausgangsmaterial verwendeten wir neben normaler Rattenleber die Tumoren Hepatom Berlin und DAENA 7330, zwei ursprünglich im Institut für Krebsforschung der DAW zu Berlin, Berlin-Buch, aus Buttergelb bzw. Diäthylnitrosamin erzeugte transplantable Rattenlebercarcinome. Die Aufarbeitung der Gewebe und ihre Fraktionierung wurde nach dem bereits beschriebenen ${ }^{3,5}$, jedoch leicht modifizierten Verfahren durchgeführt. Die Gewinnung des hochtourigen Überstandes erfolgte durch 1-stdg. Zentrifugation bei $120000 \mathrm{~g}$ und $0^{\circ}$ in der präparativen Ultrazentrifuge VAC 60 (Fa. Janetzki, Engelsdorf b. Leipzig**), die Säureextraktion mit $0,25-n \cdot \mathrm{H}_{2} \mathrm{SO}_{4}$ (Endkonzentration). Nach Dialyse gegen Wasser und 0,01-n. Essigsäure wurde der Säureextrakt im Rotationsverdampfer bei $+25^{\circ}$ eingeengt und gefriergetrocknet. Ausbeute (pro $100 \mathrm{~g}$ gewaschenes Gewebe): Leber $400-500 \mathrm{mg}$, Hepatom Berlin $600-700 \mathrm{mg}$, DAENA 500-600 mg.

5 K. D. Schwenke u. M. Kujawa, Z. Naturforschg. 22 b, 961 [1967].

*6. Mitt. s. l. c. ${ }^{5}$.

** Für die Möglichkeit zur Benutzung der Ultrazentrifuge möchte ich Herrn Dr. habil. H. KobliTz, Institut für Faserstoff-Forschung der DAW zu Berlin in Teltow-Seehof, verbindlichst danken. 